\title{
Dermoscopy of Primary Cutaneous Amyloidosis in Skin of Color
}

\author{
Feroze Kaliyadan ${ }^{1}$, Abdulaziz Alkhateeb ${ }^{1}$, Joel Kuruvilla ${ }^{1}$, \\ Krishna Swaroop², Abdulrahim A. Alabdulsalam²
}

1 Department of Dermatology, College of Medicine, King Faisal University, Al Ahsa, Saudi Arabia
2 Department of Pathology (Biomedical Sciences), College of Medicine, King Faisal University, Al Ahsa, Saudi Arabia

Key words: dermoscopy, primary cutaneous amyloidosis, high dynamic range conversion

Citation: Kaliyadan F, Alkhateeb A, Kuruvilla J, Swaroop K, Alabdulsalam AA. Dermoscopy of primary cutaneous amyloidosis in skin of color. Dermatol Pract Concept. 2019;9(3):232-234. DOI: https://doi.org/10.5826/dpc.0903a17

Accepted: December 23, 2018; Published: July 31, 2019

Copyright: $@ 2019$ Kaliyadan et al. This is an open-access article distributed under the terms of the Creative Commons Attribution License, which permits unrestricted use, distribution, and reproduction in any medium, provided the original author and source are credited.

Funding: None.

Competing interests: The authors have no conflicts of interest to disclose.

Authorship: All authors have contributed significantly to this publication.

Corresponding author: Feroze Kaliyadan, MD, DNB, MNAMS, FIMSA, SCE-RCP, Faculty of Dermatology, College of Medicine, King Faisal University, Al Ahsa, Saudi Arabia-31982. Email: ferozkal@hotmail.com

\section{Introduction}

We present a case of a patient with Fitzpatrick skin type V presenting with primary cutaneous amyloidosis, in whom polarized dermoscopy mainly showed irregular white central areas, surrounded completely or partially by thick hyperpigmented blotches.

\section{Case Presentation}

A 57-year-old woman with Fitzpatrick skin type V presented with hyperpigmented skin lesions over her body of more than 6 years' duration. The lesions were located mainly over the upper trunk and limbs and were moderately itchy (Figure 1). There was a history of previous treatment with topical corticosteroids and antihistamines. Prior to the lesions, she was regularly using loofahs and scrubs, which were stopped as per the advice of the dermatologist. On examination, areas of reticulate macular hyperpigmentation and hyperpigmented papules were seen. A clinical diagnosis of primary

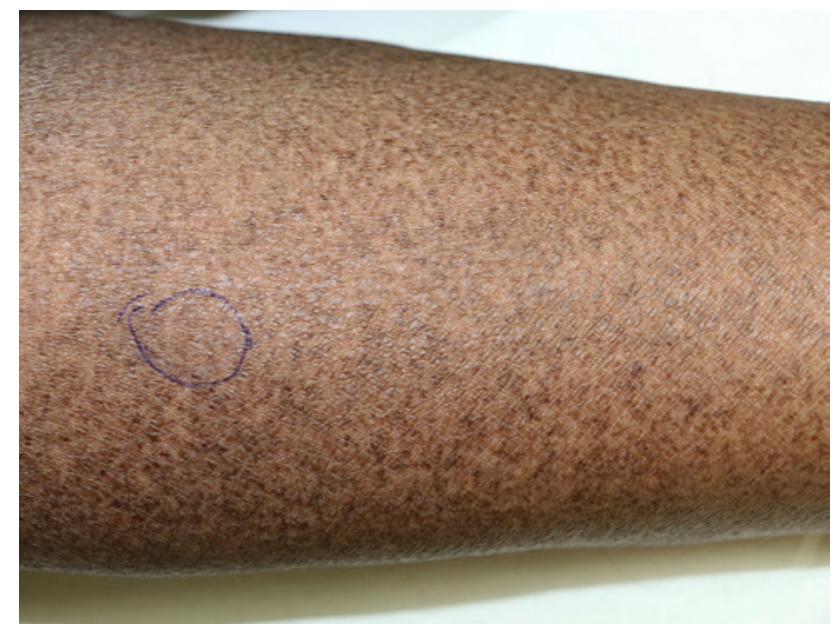

Figure 1. Hyperpigmented macules and papules over the forearm. [Copyright: @2019 Kaliyadan et al.]

amyloidosis was considered (a possibility of a biphasic amyloidosis was thought of because the lesions showed features suggestive of both macular and lichen amyloidosis 


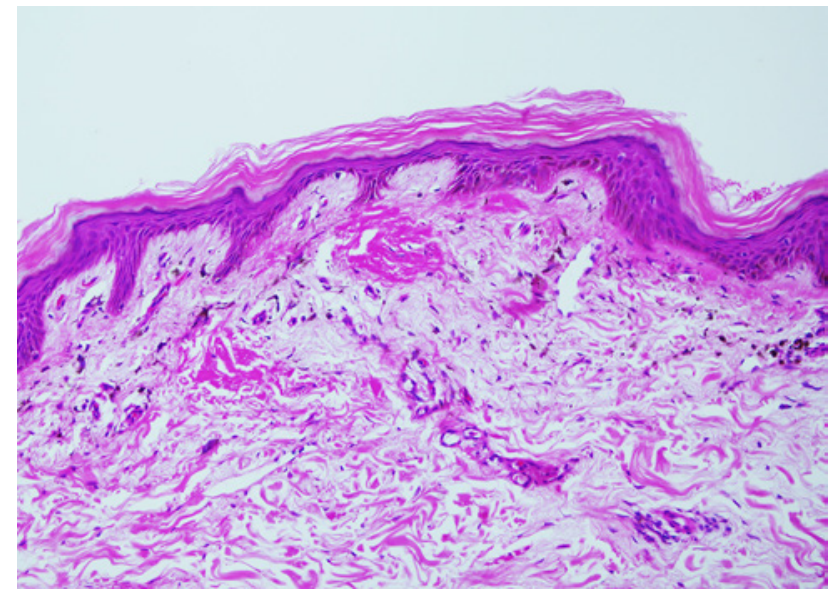

Figure 2. Histopathology shows deposits of amorphous eosinophilic material in the superficial dermis associated with numerous pigment-laden macrophages. There is minimal perivascular inflammatory infiltrate (hematoxylin and eosin, $\times 400$ ). [Copyright: (C2019 Kaliyadan et al.]

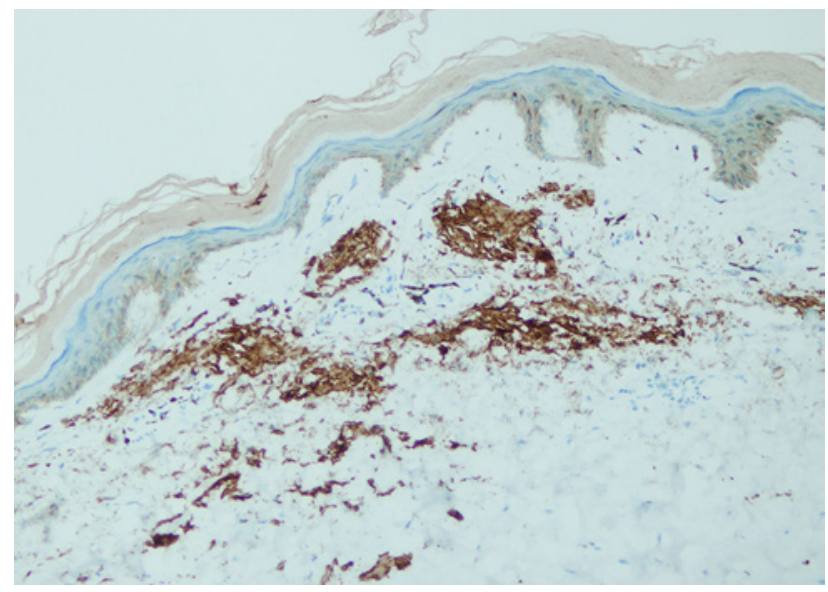

Figure 3. Multiple deposits of amorphous material in the superficial dermis, strong positive staining with cytokeratin staining (CK 34BE12, ×200). [Copyright: (2019 Kaliyadan et al.] with combination of flat and raised lesions). A skin biopsy showed deposits of amorphous eosinophilic material in the superficial dermis associated with numerous pigment-laden macrophages (Figure 2). Immunohistochemistry showed strongly positive staining with CK 34BE12 (Figure 3). Based on the clinical and histopathology findings, a diagnosis of primary cutaneous amyloidosis was confirmed.

Dermoscopy (polarized $\times 10$, DermLite Foto II Pro) showed a combination of patterns. The papular lesions showed a central white area encircled by thick brown to black pigmentation. Some of the flat lesions showed irregular white central areas, surrounded completely or partially by thick hyperpigmented blotches, while other flat lesions just showed irregular hyperpigmented blotches. Streaks were not visualized (Figure 4). The patterns were further highlighted using high dynamic range (HDR) conversion (Figure 5; Topaz filter in Adobe Photoshop CC 2015).

\section{Conclusions}

There are very few reports related to the dermoscopy of primary cutaneous amyloidosis. Chuang et al reported a study of 35 cases in which the primary patterns seen included a central hub, either white or brown, surrounded by various configurations of hyperpigmentation and for cases with prominent hyperkeratosis such as in lichen amyloidosis, the central hub was replaced by a scar-like white area [1,2]. Atypical patterns such as yellow, teardrop-like areas and orange-yellow homogeneous backgrounds with serpentine vessels have been described in cases of nodular amyloidosis [3,4]. Arnold and Bowling have reported "shiny white streaks" on polarized dermoscopy in 2 patients with lichen amyloidosis. One of the patients mentioned in this report is of "Indian ethnicity," but the patient's exact skin type was not mentioned. The author hypothesized that under polarizing dermoscopy birefringent amyloid fibrils are detected as shiny white streaks [5]. We could not find something similar in our case.

We suggest that HDR conversion is a useful tool in accentuating pigmentary patterns on dermoscopy. HDR has been described to improve visualization of dermoscopy patterns in the context of skin tumors [6]. We have done some work in the use of HDR in vitiligo (in print) and believe that it is a useful tool to enhance visualization of patterns in the context of pigmentary disorders in

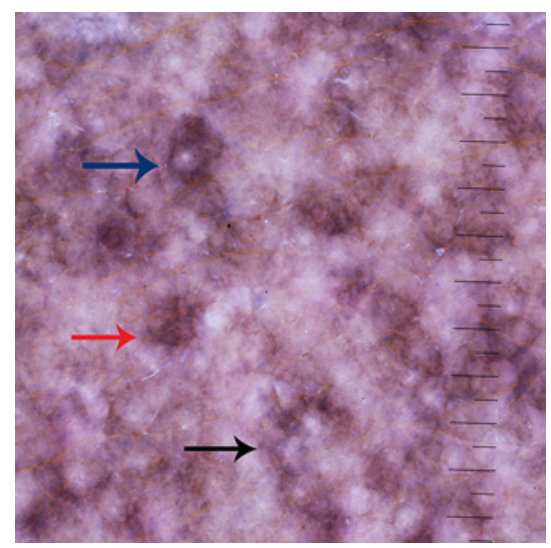

Figure 4. The papular lesions show a central white area encircled by thick brown to black pigmentation (blue arrow). Some of the flat lesions show irregular white central areas, surrounded completely or partially by thick hyperpigmented blotches (black arrow), while other flat lesions just show irregular hyperpigmented blotches (red arrow). [Copyright: @2019 Kaliyadan et al.]

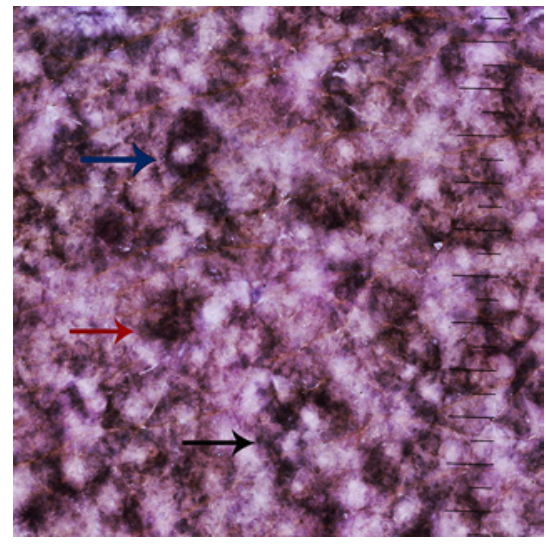

Figure 5. HDR conversion of Figure 4 showing accentuation of the pigmentary patterns. [Copyright: (C2019 Kaliyadan et al.] 
general. While in this report we use single-image conversion using a specific software, most digital cameras nowadays, including mobile phones, have in-built HDR conversion mechanisms, which can be used to improve the quality of dermoscopy images in general.

Although we realize that significant conclusions cannot be made from a single case, we propose that the dermoscopy patterns for primary cutaneous amyloidosis are likely to be different in patients with skin of color. While we did see areas corresponding to the pattern described by Chuang et al in the form of white hubs surrounded by different patterns of hyperpigmentation, the specific morphology and amount of pigmentation was different. We did not see specific patterns like venation or streaks. In addition, the pigmentation appeared to be thicker and darker in our case, which we believe could correlate with the darker skin type.

\section{References}

1. Chuang YY, Lee DD, Lin CS, et al. Characteristic dermoscopic features of primary cutaneous amyloidosis: a study of 35 cases. $\mathrm{Br}$ J Dermatol. 2012;167(3):548-554.

2. Moscarella E, Ronchi A, Agozzino M, Franco R, Argenziano G. Image Gallery: Dermoscopy of lichen amyloidosis. $\mathrm{Br} J$ Dermatol. 2018;179(6):e231.

3. Rongioletti F, Atzori L, Ferreli C, Pinna A, Aste N, Pau M. A unique dermoscopy pattern of primary cutaneous nodular amyloidosis mimicking a granulomatous disease. J Am Acad Dermatol. 2016;74(1):e9-e10.

4. Di Meo N, Noal C, Fadel M, Trevisan G. Yellow teardrop-like structures in primary nodular skin amyloidosis. G Ital Dermatol Venereol. 2018;153(1):118-119.

5. Arnold SJ, Bowling JC. "Shiny white streaks" in lichen amyloidosis: a clue to diagnosis. Australas J Dermatol. 2012;53(4):272-273.

6. Braun RP, Marghoob A. High-dynamic-range dermoscopy imaging and diagnosis of hypopigmented skin cancers. JAMA Dermatol. $2015 ; 151(4): 456-457$. 